\section{Neue ÄDA-Mitgliedsbeiträge 2012}

A uf Beschluss des Vorstandes wurden die Mitgliedsbeiträge im Ärzteverband Deutscher Allergologen (ÄDA) ab 2012 folgendermaßen geändert:

_Der reguläre Mitgliedsbeitrag - inklusive Allergo Journal (acht Hefte pro Jahr) - beträgt $265 €$.

_ Für nicht liquidationsberechtigte Ärzte gilt der reduzierte Beitrag - ebenfalls inklusive Allergo Journal (acht Hefte

—Gänzlich vom Beitrag befreit sind Juniormitglieder bis zum 36. Geburtstag und Mitglieder nach Neugründung einer Praxis für zwei Jahre. Diese Mitglieder Journal . pro Jahr) von $135 €$.

erhalten ebenfalls Zugang zum Allergo

Für die Sondertarife wird ein Nachweis erbeten. Eine Doppelmitgliedschaft ÄDA/ DGAKI (Deutsche Gesellschaft für Allergologie und klinische Immunologie) wird bei der DGAKI berücksichtigt. Die Kongressgebühren für den jährlich stattfindenden Deutschen Allergiekongress sind für Mitglieder des ÄDA deutlich erniedrigt.

Den hier abgedruckten Aufnahmeantrag finden Sie auf der linken Seite sowie als PDF-Datei zum Herunterladen auf der ÄDA-Homepage unter www.aeda.de.

Prof. Dr. Ludger Klimek

\section{ÄDA-Website - aktualisiert und optimiert}

D ie neue Internetseite des Ärzteverbands Deutscher Allergologen (ÄDA) ist online, unter www.aeda.de. Die zeitgemäße Technik des OnlineAngebots sowie die moderne Gestaltung im neuen ÄDA-Design erleichtern nun die Orientierung der Besucher, die mit Hilfe der neu entwickelten Navigation einfach und schnell ans gewünschte Ziel kommen.

Auf der Startseite werden in einem animierten Banner besonders wichtige Mitteilungen optisch stark hervorgehoben. Darunter finden sich Nachrichten aus Beruf und Verband, die laufend ergänzt und aktualisiert werden.

In der rechten Randspalte sind jeweils kurzfristig anstehende Veranstaltungen zu sehen. Direkt darunter führt ein Link zu einer ausführlichen Veranstaltungsliste, zu der jedermann eigene passende Veranstaltungen über die ÄDA-Geschäftsstelle anmelden kann.

Ein wichtiges Element für Patienten ist die ebenfalls in der rechten Randspalte platzierte Allergologensuche. Da die Kassenärztlichen Vereinigungen Allergologen nicht separat ausweisen, bietet ausschließlich die ÄDA-Website die Möglichkeit, in Deutschland systematisch nach Ärzten mit einer Spezialisierung auf Allergologie zu suchen.

In einem passwortgeschützten Bereich haben ÄDA-Mitglieder Zugang zu besonderen Diensten und Leistungen. Daneben

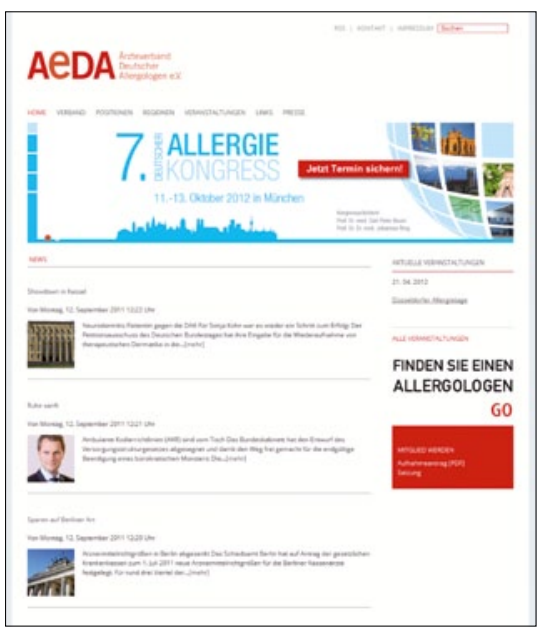

gibt es für alle Besucher Informationen rund um den Verband sowie seine Leistungen, eine umfangreiche Link-Liste, die vor allem Links enthält, die man nicht mit Hilfe eines oder zweier Suchwörtern bei den einschlägigen Suchmaschinen findet, einen Bereich mit Mitteilungen für die Presse und Wissenswertes rund um die Allergologie.

Eine gut funktionierende Suche, die ganz oben rechts auf der Website platziert ist, erleichtert das Auffinden von Informationen auf direktem Wege.

Der ÄDA wird seinen Internet-Auftritt künftig laufend weiter entwickeln und $\mathrm{zu}$ einer zentralen Anlaufstelle für Informationen zur Allergologie ausbauen.

Ulrich Glatzer

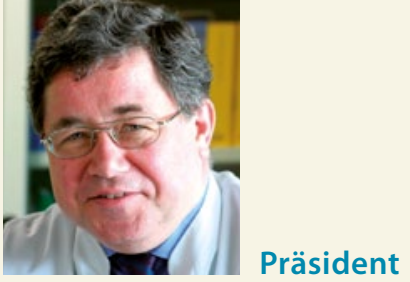

Prof. Dr. Hans F. Merk

Hautklinik der Medizinischen Fakultät, Uniklinikum der RWTH, Aachen

E-Mail: hans.merk@post.rwth-aachen.de

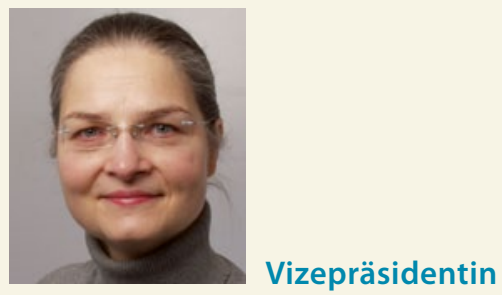

Priv-Doz. Dr. Kirsten Jung Praxis für Dermatologie und Immunologie, Erfurt

E-Mail: kirsten-jung@t-online.de

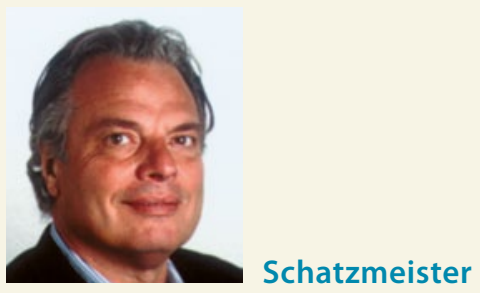

Prof. Dr. Wolfgang W. Schlenter Klinik für HNO-Krankheiten, St. Marienkrankenhaus, Katharina-Kasper-Kliniken, Frankfurt/Main

E-Mail:w.schlenter@katharina-kasper.de

\section{Beisitzer}

_Prof. Dr. Wolfgang Czech, VS-Villingen

_Dr. Frank Friedrichs. Aachen

_Prof. Dr. Thomas Fuchs, Göttingen

_Prof. Dr. Ludger Klimek, Wiesbaden

_Prof. Dr. Wolfgang Leupold, Dresden

_Norbert K. Mülleneisen, Leverkusen

_Dr. Uta Rabe, Treuenbrietzen

_Prof. Dr. Wolfgang Wehrmann, Münster

\section{Geschäftsstelle}

Service Systems

Carin Fresle und Ursula Raab

Blumenstraße 14

63303 Dreieich

Tel.: (0 61 03) 62273 und

(0 6103$) 63657$

Fax: (0 61 03) 697019

E-Mail: info@aeda.de 